\title{
Hypoxia-inducible factor- $1 \alpha$ promotes endometrial stromal cells migration and invasion by upregulating autophagy in endometriosis
}

\author{
Hengwei Liu ${ }^{1}$, Zhibing Zhang ${ }^{2}$, Wenqian Xiong ${ }^{1}$, Ling Zhang ${ }^{1}$, Yao Xiong ${ }^{1}, \mathrm{Na} \mathrm{Li}^{1}$, Haitang He${ }^{1}$, \\ Yu Du ${ }^{1}$ and Yi Liu ${ }^{1}$ \\ ${ }^{1}$ Department of Obstetrics and Gynecology, Union Hospital, Tongji Medical College, Huazhong University of \\ Science and Technology, Wuhan, China and ${ }^{2}$ Department of Obstetrics and Gynecology, Virginia Commonwealth \\ University, Richmond, Virginia, USA
}

Correspondence should be addressed to Y Liu; Email: Liqun94@163.com

\begin{abstract}
Endometriosis is a benign gynecological disease that shares some characteristics with malignancy like migration and invasion. It has been reported that both hypoxia-inducible factor-1 $\alpha$ (HIF-1 $\alpha$ ) and autophagy were upregulated in ectopic endometrium of patients with ovarian endometriosis. However, the crosstalk between HIF-1 $\alpha$ and autophagy in the pathogenesis of endometriosis remains to be clarified. Accordingly, we investigated whether autophagy was regulated by HIF-1 $\alpha$, as well as whether the effect of HIF-1 $\alpha$ on cell migration and invasion is mediated through autophagy upregulation. Here, we found that ectopic endometrium from patients with endometriosis highly expressed HIF-1 $\alpha$ and autophagy-related protein LC3. In cultured human endometrial stromal cells (HESCs), autophagy was induced by hypoxia in a time-dependent manner and autophagy activation was dependent on HIF-1 $\alpha$. In addition, migration and invasion ability of HESCs were enhanced by hypoxia treatment, whereas knockdown of HIF-1 $\alpha$ attenuated this effect. Furthermore, inhibiting autophagy with specific inhibitors and Beclin1 siRNA attenuated hypoxia triggered migration and invasion of HESCs. Taken together, these results suggest that HIF-1 $\alpha$ promotes HESCs invasion and metastasis by upregulating autophagy. Thus, autophagy may be involved in the pathogenesis of endometriosis and inhibition of autophagy might be a novel therapeutic approach to the treatment of endometriosis.

Reproduction (2017) 153 809-820
\end{abstract}

\section{Introduction}

Endometriosis, a common contributor for infertility and chronic pelvic pain, is characterized by the extrauterine growth of endometrial glands and stroma (Giudice \& Kao 2004). Among the numerous theories regarding the pathogenesis of endometriosis, the most commonly accepted one is Sampson's hypothesis of retrograde menstruation, which states that the endometrial tissues shed from uterine cavity during menses exit the uterus through fallopian tubes (Sampson 1927). Although endometriosis is generally assumed to be a benign disease, it has the similar malignant biological behavior like cancer, such as aggressive migration and invasion (Bassi et al. 2009), which is crucial for the development of endometriosis.

Accumulating evidence has suggested that hypoxia played important roles in endometriosis (Lin et al. 2012, Hsiao et al. 2014). Hypoxia-inducible factor (HIF)-1, a heterodimeric transcriptional factor mediating the cellular response to hypoxia, is made up of $\alpha$ and $\beta$ subunits (Majmundar et al. 2010). Unlike the constitutively expressed HIF-1 $\beta$, HIF- $1 \alpha$ is highly regulated by cellular oxygen tension. Under normoxic conditions, the HIF$1 \alpha$ subunit is rapidly degraded; whereas under hypoxic conditions, HIF- $1 \alpha$ is stabilized and translocated to the nucleus where it heterodimerized with HIF- $1 \beta$ to cause target gene transcription (Semenza 2009). HIFmodulated genes have been identified to contribute to invasion, angiogenesis and autophagy of different types of tumor cells (Zhao et al. 2010, Cheng et al. 2013). Moreover, the expression level of HIF- $1 \alpha$ in serum and ectopic endometrium of patients with endometriosis was elevated (Wu et al. 2007, Karakus et al. 2016).

Autophagy is an evolutionary conserved process responsible for the bulk degradation of cytoplasmic components, such as long-lived proteins and damaged organelles (Hale et al. 2013). During autophagy, double-membrane vesicles surround and deliver the cytoplasmic material to lysosomes for degradation (Yang \& Klionsky 2010). Autophagosome formation requires two ubiquitin-like protein conjugation pathways: autophagy-related gene (Atg)12-Atg5 conjugation 
system and the microtubule-associated protein light chain 3 beta (LC3)-lipid phosphatidylethanolamine (PE) conjugation system (Ohsumi \& Mizushima 2004). LC3, a mammalian homolog of yeast Atg8, is known to be present on autophagosomes, and the conversion of LC3 I-II is a widely used marker to monitor this process (Mizushima et al. 2010). Beclin1 (also named ATG6) is another key regulator, which plays essential roles in autophagy activation (Kang et al. 2011). It is now generally accepted that autophagy is a protective mechanism for cells adaptation to stress conditions like hypoxia (Bhogal et al. 2012, Hu et al. 2012). Interestingly, autophagy upregulation was observed in ectopic endometrium of patients with ovarian endometriosis (Allavena et al. 2015). However, it is still unclear whether autophagy was activated in human endometrial stromal cells (HESCs) under hypoxia environment, and few studies have elucidated the correlation between HIF- $1 \alpha$ and autophagy in the pathogenesis of endometriosis.

Therefore, in the present study, we aimed to investigate (a) whether the expression levels of HIF-1 $\alpha$ and autophagy were changed in ectopic endometrium; (b) the molecular mechanism of autophagy activation by hypoxia in human endometrial stromal cells (HESCs) and (c) the possible role of autophagy in HIF- $1 \alpha$-induced migration and invasion of HESCs.

\section{Materials and methods \\ Ethical approval}

The tissue samples were obtained with full and informed patient consent. Ethics approval for this study was obtained from the Local Ethics Committee of Tongji Medical College, Huazhong University of Science (IORG No: IORG0003571).

\section{Patients and tissue collection}

The patients recruited for the study were non-pregnant women of childbearing age (22-48 years) attending the Department of Obstetrics and Gynecology, Union Hospital, Tongji Medical College, Huazhong University of Science and Technology between October 2013 and October 2015. All patients had regular menstrual cycles and were not taking any combination hormonal contraception for at least six months prior to surgery. The endometrial samples were collected during the proliferative stage, which was confirmed based on clinical or histologic criteria.

As controls, eleven cases of normal endometrium were obtained from patients with tubal infertility. Ten cases of eutopic endometrium (from another group of women with ovarian endometriosis) and ten cases of ectopic endometrium (from ovarian endometriotic cysts) were obtained from patients who underwent laparoscopic surgery or hysterectomy. All of the ectopic endometrium were classified as revised American Fertility Society stage III or IV (1997). The collected endometrial tissues was divided into two parts: the first part was used for immunohistochemistry (IHC) analysis according to the criteria of Noyes and coworkers (Noyes et al. 1975), and the second part was used for Western blot analysis. Besides, another thirty cases of eutopic endometrium of patients with endometriosis were collected for isolation and cultivation of endometrial stromal cells. The endometrial tissues were collected using the Nowak's curette just before the surgical procedure and immediately transported to the laboratory.

\section{Immunohistochemistry}

All fresh surgical specimens were fixed in 10\% formaldehyde for $24 \mathrm{~h}$, and then embedded in paraffin blocks. Formalin-fixed and paraffin-embedded endometrial tissues were sectioned at $5 \mu \mathrm{m}$ and mounted on alcohol-cleaned glass slides. The sections were dewaxed in xylene and rehydrated by passing through a graded series of alcohol to water, and antigen retrieval was performed by heating sections in citrate buffer at $\mathrm{pH}$ 6.0. Endogenous non-specific peroxidase activity was quenched by incubating the section in 50\% ethanol solution containing $3 \% \mathrm{H}_{2} \mathrm{O}_{2}$ for $30 \mathrm{~min}$. The sections were sequentially blocked with protein block for $30 \mathrm{~min}$ followed by blocking in bovine serum albumin for $30 \mathrm{~min}$ and then incubated with primary antibodies against HIF-1 $\alpha$ (1:1000; Affinity, Cincinnati, $\mathrm{OH}$, USA) and LC3B (1:1000; Abcam) overnight at $4^{\circ} \mathrm{C}$. After washing in PBS, the sections were incubated with peroxidaselabeled anti-rabbit IgG (1:500; Wuhan Boster Biotechnology Co. Ltd, Wuhan, China) for $30 \mathrm{~min}$. Finally, all slides were incubated with DAB-Substrate (Beyotime, Shanghai, China) and counterstained in hematoxylin before dehydration and mounted. After the immunohistochemical analysis, IPP software (image-pro plus 6.0) was used to analyze the optical density of the representative images (Supplementary Table 1, see section on supplementary data given at the end of this article).

\section{Isolation and culture of human endometrial stromal cells (HESCs)}

The collected tissues were washed with PBS for three times, and then minced into $1 \mathrm{~mm}$ pieces with sterile surgical scissors and digested in PBS containing $2 \mathrm{mg} / \mathrm{mL}$ of type II collagenase $\left(0.1 \%\right.$, Sigma-Aldrich) at $37^{\circ} \mathrm{C}$ for $45-60 \mathrm{~min}$ with constant agitation. Stromal cells were isolated from the epithelial cells and debris using 150 and $37.4 \mu \mathrm{m}$ sieves, and the filtered stromal cells were plated in T25 flasks. After overnight culture, the stromal cells attached, and the contaminated blood cells and debris that were suspended in the culture medium were removed by aspiration and the stromal cells were washed with PBS. The stromal cells were subsequently cultured in Dulbecco's modified Eagle's/F12 medium (DMEM/F12; HyClone) and supplemented with 20\% fetal bovine serum (FBS; HyClone), $100 \mathrm{U} / \mathrm{mL}$ penicillin and $100 \mathrm{mg} / \mathrm{mL}$ streptomycin (HyClone) in humidified atmosphere with $5 \% \mathrm{CO}_{2}$ at $37^{\circ} \mathrm{C}$. The purity of isolated stromal cells was $>95 \%$, and stromal cells were contaminated by less than $1 \%$ of epithelial cells, as determined by diffuse and strong cytoplasmic immunostaining for vimentin (diluted 1:100; Cell Signaling Technology) and negative cellular staining for E-cadherin (diluted 1:150; Cell Signaling Technology) in immunocytochemistry (Supplementary Fig. 1). Endometrial stromal cells were 
cultured in DMEM/F12 medium with the addition of either $10 \mathrm{mM} 3$-methyladenine (3-MA) or $500 \mu \mathrm{M}$ chloroquine to inhibit autophagy.

\section{Hypoxia treatment}

Upon reaching confluence, the endometrial stromal cells $\left(4 \times 10^{5}\right)$ were seeded in $60 \mathrm{~mm}$ culture dishes, and fresh medium was used to keep the cells healthy by providing fresh nutrients before hypoxia treatment. The culture dishes were incubated in a modular incubator chamber (Thermo Fisher Scientific) containing humidified hypoxic air $\left(1 \% \mathrm{O}_{2}, 5 \% \mathrm{CO}_{2}\right.$ and $94 \% \mathrm{~N}_{2}$ ) for $0,4,8,16$ and $24 \mathrm{~h}$ at $37^{\circ} \mathrm{C}$. Stromal cells cultured under normoxic condition $\left(20 \% \mathrm{O}_{2}, 5 \% \mathrm{CO}_{2}\right.$ and $75 \% \mathrm{~N}_{2}$ ) were used as controls.

Hypoxia-treated cells were collected at the indicated time points and prepared for Western blot analysis. After culture under hypoxic conditions for 24h, monodansylcadaverine (MDC) staining and acridine orange (AO) staining were performed to detect the accumulation of autophagic vacuoles. In addition, the ultrastructure of autophagosomes in hypoxiatreated cells was observed by transmission electron microscopy.

\section{Immunocytochemical staining of HESCS}

The immunocytochemistry was performed to detect mesenchymal marker vimentin and epithelial marker E-cadherin. HESCs were plated into a 6 -well plate at a density of $2 \times 10^{4}$ cells/well and grown until approximately $80 \%$ confluent. The medium was removed, and the cells were washed three times in PBS. After fixation with $4 \%$ paraformaldehyde at $4^{\circ} \mathrm{C}$ for $15 \mathrm{~min}$, the cells were soaked in $0.3 \%$ Triton X-100 (Sigma) for $15 \mathrm{~min}$ to increase their permeability to antibodies. For blocking unspecific binding site of antigens, the cells were rinsed with $10 \%$ bovine serum albumin in PBS for $60 \mathrm{~min}$, and incubated overnight at $4{ }^{\circ} \mathrm{C}$ with the primary antibodies mentioned previously. The cells were then washed three times in PBS and incubated with horseradish peroxidase-conjugated secondary antibody for two hours, followed by washing in PBS. Images were collected using an Eclipse TE2000-S microscope system (Nikon UK) and Image-Pro Plus (Media Cybernetics UK, Berkshire, UK).

\section{Protein extraction and Western Blot analysis}

Collected endometrial tissues and cultured HESCs were washed three times with ice-cold PBS and lysed in radio immunoprecipitation assay (RIPA) buffer (Beyotime Biotechnology, China) containing protease inhibitors (Sigma). The cells were scraped in this lysis buffer, kept on ice for at least $30 \mathrm{~min}$, centrifuged at $12,000 \mathrm{~g}$ at $4{ }^{\circ} \mathrm{C}$ for $15 \mathrm{~min}$ and diluted in $5 \times$ sample buffer (Beyotime Biotechnology). BCA protein assay kit (Beyotime) was used to determine the protein concentrations. Equal amounts of proteins $(30 \mu \mathrm{g})$ were mixed with the sample buffer (4\% SDS, 10\% beta-mercaptoethanol and $20 \%$ glycerol in $0.125 \mathrm{M}$ Tris, $\mathrm{pH}$ 6.8) containing bromophenol blue and were boiled for $10 \mathrm{~min}$ at $95^{\circ} \mathrm{C}$. The samples were loaded and separated by $12 \%$ sodium dodecyl sulfate-polyacrylamide gel electrophoresis gels (PAGE) with running buffer. The proteins separated by SDS-PAGE were transferred to polyvinylidene difluoride (PVDF) membranes (Immobilon-P transfer membrane). The membranes were incubated with $5 \%$ fat-free milk in Tris-buffered saline containing $0.05 \%$ Tween 20 for $1 \mathrm{~h}$ and were then incubated overnight at $4{ }^{\circ} \mathrm{C}$ with the following primary antibodies: HIF$1 \alpha$ (diluted 1:1000; Affinity), LC3B (diluted 1:1000, Abcam), Beclin1 (diluted 1:1000, Abcam) and GAPDH (diluted 1:1000; Affinity). The membranes were washed three times with TBST for $15 \mathrm{~min}$, and then incubated with an HRP-labeled secondary $\mathrm{Ab}$ at room temperature for $1 \mathrm{~h}$. The membranes were washed again and treated with ECL-Western blot-detecting reagent (Millipore) according to the manufacturer's recommendations. The protein bands intensity were observed by imaging system (Gel Doc 2000; Bio-Rad) and analysis with ImageJ software (NIH) (version 1.5).

\section{Acridine orange (AO) staining assay}

Acridine orange (AO) is a fluorescent cationic dye used to detect acidic vesicular organelles (lysosomes) within cells. It can interact with DNA emitting green fluorescence or accumulate in acidic organelles in which it becomes protonated forming aggregates that emit bright yellow-toorange fluorescence (Pierzynska-Mach et al. 2014). The cytoplasm and nucleus showed bright green fluorescent signal, whereas the acidic vesicular organelles showed bright yellowto-orange fluorescent signal. Briefly, $5 \times 10^{4}$ cells were stained with $1 \mu \mathrm{g} / \mathrm{mL}$ acridine orange (AO) (Sigma-Aldrich) in PBS and incubated for $15 \mathrm{~min}$ at $37^{\circ} \mathrm{C}$ in the darkness. After incubation, cells were washed with PBS for three times and immediately observed using an inverted fluorescence microscope (IX51, Olympus). The autophagy was measured by quantification of the rate of AO-positive stained vacuoles in five random fields (a field containing at least 40 cells) for each experimental condition.

\section{Monodansylcadaverine (MDC) staining assay}

To detect the autophagic vacuoles, monodansylcadaverine (MDC), a fluorescent dye known as specific marker for autophagic vacuoles, was used. $5 \times 10^{4}$ cells were grown on coverslips in 6-well plate and cultured under hypoxic conditions for the indicated time, followed by washing three times with PBS and fixed in $10 \%$ formalin solution for $10 \mathrm{~min}$. Then, cells were stained with $0.05 \mathrm{mM}$ MDC (Sigma-Aldrich) for $15 \mathrm{~min}$ at $37^{\circ} \mathrm{C}$ in the darkness. The following procedures were the same as AO staining. The autophagy was measured by quantification of the rate of MDC positive-stained vacuoles in five random fields (a field containing at least 40 cells) for each experimental condition.

\section{Transmission electron microscopy (TEM)}

To identify autophagosomes at the ultrastructural level, HESCs were cultured under hypoxic or normoxic conditions for $24 \mathrm{~h}$. After the indicated treatment, HESCs were washed three times with PBS and incubated with trypsin for $2 \mathrm{~min}$. Cells were collected by centrifugation at $1000 \mathrm{~g}$ for $5 \mathrm{~min}$. The cell 
pellets were suspended and fixed with $2.5 \%$ glutaraldehyde in $0.1 \mathrm{M} \mathrm{Na}$-phosphate buffer $(\mathrm{pH} 7.4)$ at $4^{\circ} \mathrm{C}$ overnight, and then washed in $0.1 \mathrm{M} \mathrm{Na}$-phosphate buffer $(\mathrm{pH}$ 7.4) twice for $15 \mathrm{~min}$ each and post-fixed with $1 \% \mathrm{OsO}_{4}$ in $0.1 \mathrm{M}$ cacodylate buffer ( $\mathrm{pH}$ 7.4) for $3 \mathrm{~h}$. After being washed by $0.1 \mathrm{M} \mathrm{Na}-$ phosphate buffer, the cells were then dehydrated at $25^{\circ} \mathrm{C}$ with a graded series of ethanol and gradually infiltrated with epoxy resin mixture (812 resin embedding kit). The samples were sequentially polymerized at $37^{\circ} \mathrm{C}$ for $12 \mathrm{~h}, 45^{\circ} \mathrm{C}$ for $12 \mathrm{~h}$, and $60^{\circ} \mathrm{C}$ for $24 \mathrm{~h}$. Ultrathin sections $(50-70 \mathrm{~nm})$ were cut using LKB microtome and mounted on single-slot copper grids. THE sections were subjected to double staining with uranyl acetate and lead citrate and examined using a transmission electron microscope (Philips CM-120).

\section{Cell transfection assay}

HIF-1 $\alpha$ siRNA, Beclin1 siRNA and scrambled negative control siRNA were purchased from Shanghai GenePharma (China). The siRNA sequences included HIF-1 $\alpha$ siRNA (sense, 5'-GCUGGAGACAAUCAUAUTT-3', antisense, 5'-AUAUGAUUGUGUCUCCAGCTT-3'), Beclin1 SIRNA (sense, 5'-CGGGAAUACAGUGAAUUUATT-3', antisense, $\quad 5^{\prime}$-UAAAUUCACUGUAUUCCCGTT-3') and scrambled negative control siRNA (sense, 5'-UUCUCCGAACGUGUCACGUTT-3'; antisense, 5'-ACGUGACACGUUCGGAGAATT-3'). HIF-1 $\alpha$ overexpression plasmid (pG/CMV/HIF-1 $\alpha /$ IRES/EGFP) and negative control (NC) plasmid were purchased from Gemma Pharmaceutical Technology (China). For knockdown, HESCs $\left(2 \times 10^{5}\right.$ cells/well) were seeded in 6-well plates and grown to $60-80 \%$ confluence, followed by transfection with the previously mentioned plasmids or siRNA using Lipofectamine2000 (Invitrogen, Life Technologies) according to the manufacturer's protocol. Transfection mixture was replaced $6 \mathrm{~h}$ later with DMEM/F-12 with $20 \%$ FBS. Then, HESCs were incubated in normoxic or hypoxic conditions for another $24 \mathrm{~h}$ and subjected to Western blot analysis and GFP-LC3 adenoviral vector transfection.

\section{Autophagy detection using GFP-LC3 adenoviral vector}

The indicated cells were seeded on coverslips in 24-well plates and allowed to reach $50-70 \%$ confluence at the time of transfection. GFP-LC3 adenoviral vectors were purchased from Beyotime Biotechnology Co. Ltd. Adenoviral infection was performed according to the manufacturer's instructions. HESCs were incubated in growth medium with the adenoviruses at a $\mathrm{MOI}$ of 50 for $24 \mathrm{~h}$ at $37^{\circ} \mathrm{C}$. The cells in the control group and HIF- $1 \alpha$ overexpression group were cultured under normoxic condition for another $24 \mathrm{~h}$; the cells in the siHIF-1 $\alpha$ and hypoxia groups were cultured under hypoxic condition for another $24 \mathrm{~h}$. After treatment, cells were washed with ice-cold PBS for three times and fixed with $4 \%$ paraformaldehyde for $15 \mathrm{~min}$ at room temperature. Then, the cells were washed three times with PBS and cover slips were mounted on the slides. Autophagy was immediately observed using a laser scanning confocal microscope (Olympus America). Autophagic level was determined by evaluating the number of GFP-LC3 puncta (puncta/cell were counted).

\section{Immunofluorescence assay}

The indicated cells were seeded and grown on coverslips in 6-well plates. The cells in the control group and HIF$1 \alpha$ overexpression group were cultured under normoxic condition for $24 \mathrm{~h}$; the cells in the siHIF-1 $\alpha$ and hypoxia groups were cultured under hypoxic condition for $24 \mathrm{~h}$. After treatment, cells were washed with ice-cold PBS and fixed with $4 \%$ paraformaldehyde for $15 \mathrm{~min}$. Then, cells were incubated with $5 \%$ BSA (bovine serum albumin) for $1 \mathrm{~h}$ to block nonspecific binding at room temperature and incubated with a LC3B antibody (1:300; Abcam) at $4^{\circ} \mathrm{C}$ overnight. The next day, the cells were incubated with goat FITC-conjugated anti-rabbit $\lg \mathrm{G}(1: 100, \mathrm{Abcam})$ for $2 \mathrm{~h}$ at dark room and then incubated with 4',6-diamidino-2 phenylindole (DAPI) for $15 \mathrm{~min}$ at room temperature. Finally, the cells were washed three times with PBS, and immediately observed using a laser scanning confocal microscope (Olympus America).

\section{Transwell migration and invasion assays}

Migration and invasion assays were performed using transwell 24-well plates with 8- $\mu \mathrm{m}$ diameter filters (Corning Costar). For invasion assay, microfilters were precoated with $40 \mu \mathrm{L}$ of working matrigel (1:3 diluted with FBS-free DMEM) (Becton, Dickinson and Company, San Diego, CA, USA) and were maintained at $37^{\circ} \mathrm{C}$ for at least $5 \mathrm{~h}$. The following procedures were the same for migration and invasion assays. Approximately $2 \times 10^{5}$ cells in $200 \mu \mathrm{L}$ of serum-free medium containing $500 \mu \mathrm{M}$ chloroquine or $10 \mathrm{mM} 3$-methyladenine (3-MA) were loaded in the upper matrigel-coated chamber and $500 \mu \mathrm{L}$ of medium containing 20\% fetal bovine serum was placed in the lower chamber. The cells cultured in normoxic condition were used as the control groups. The cells cultured under hypoxic condition with or without $500 \mu \mathrm{M}$ chloroquine or $10 \mathrm{mM} 3-\mathrm{MA}$ were used as experimental groups. To evaluate the migration potential, cells were allowed to migrate toward medium over a period of $24 \mathrm{~h}$. For the invasion assay, after seeding, cells were allowed to invade for $48 \mathrm{~h}$. After the indicated treatment, cells were fixed in methanol for $20 \mathrm{~min}$ and stained with $0.1 \%$ crystal violet for another $20 \mathrm{~min}$. Then, the cells on the upper surface of the filters were wiped off with cotton swabs, and the filters were washed three times with PBS. The cells on the underside of the filters were observed and counted under an inverted microscope at $\times 200$ magnification. Duplicate wells per condition were tested in three independent experiments.

\section{Statistical analysis}

Statistical analysis software GraphPad Prism (version 6.01; GraphPad Software) was used to carry out the statistical analyses. The Kruskal-Wallis test was used for statistical significance of differences in variables with non-normal distribution. The Student's $t$ test and one-way analysis of variance followed by Tukey's post hoc test were used to measure the comparisons between groups in normal distribution. All data sets were 
shown as mean \pm standard deviations (s.D.) from at least three independent experiments. Differences with $P$ values of $<0.05$ were considered statistically significant.

\section{Results}

The expression levels of HIF1 $\alpha$ and LC3 protein were elevated in ectopic endometrium of endometriosis patients

To determine the autophagy activity in endometriosis tissues and its relationship with HIF-1 $\alpha$, immunohistochemical staining was performed to detect the expression of HIF- $1 \alpha$ and autophagy marker LC3. Representative staining examples are shown in Fig. 1 and immunostaining score is depicted in Supplementary Table 1. As shown in Fig. 1A, HIF-1 $\alpha$ was expressed in the nuclei of epithelial and stromal cells, whereas LC3 was localized within the cytoplasm of both cells. The expression levels of HIF- $1 \alpha$ and LC3 in the ectopic endometrium were significantly greater than those in normal endometrium and eutopic endometrium from women with endometriosis. HIF- $1 \alpha$ expression levels in ectopic endometrium significantly correlated with the levels of LC3 (Supplementary Table 1). Moreover, Western blot analysis revealed a similar trend for HIF- $1 \alpha$ and LC3-II proteins expression (Fig. 1B and C). However, no significant difference was observed between normal endometrium and eutopic endometrium from the patients with endometriosis (Fig. 1B and C). Taken together, these results suggested that autophagy is upregulated in ectopic endometrium, and HIF- $1 \alpha$ may play a vital role in this event.

\section{Hypoxia-induced autophagy in HESCs}

To determine whether autophagy is activated by hypoxia stress, we performed a series of investigations to evaluate it. At first, acridine orange (AO) and monodansylcadaverine (MDC) staining were used to detect acidic vesicular organelles (AVOs), reflecting autophagosomes. As shown in Fig. 2A and B, exposure of HESCs to hypoxic conditions resulted in the accumulation of autophagic vacuoles in a timedependent manner. Electron microscopy remains to be one of the most accurate methods to detect autophagy and quantify autophagic accumulation (Swanlund et al. 2010). The ultrastructural results showed that numerous cytoplasmic phagolysosomes were present after hypoxia treatment (Fig. 2C, right panel). However, few phagolysosomes was observed in the control group (Fig. 2C, left panel). Furthermore, Western blot
A

Normal endometrium

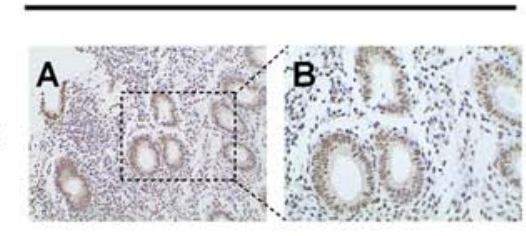

HIF1a

LC3
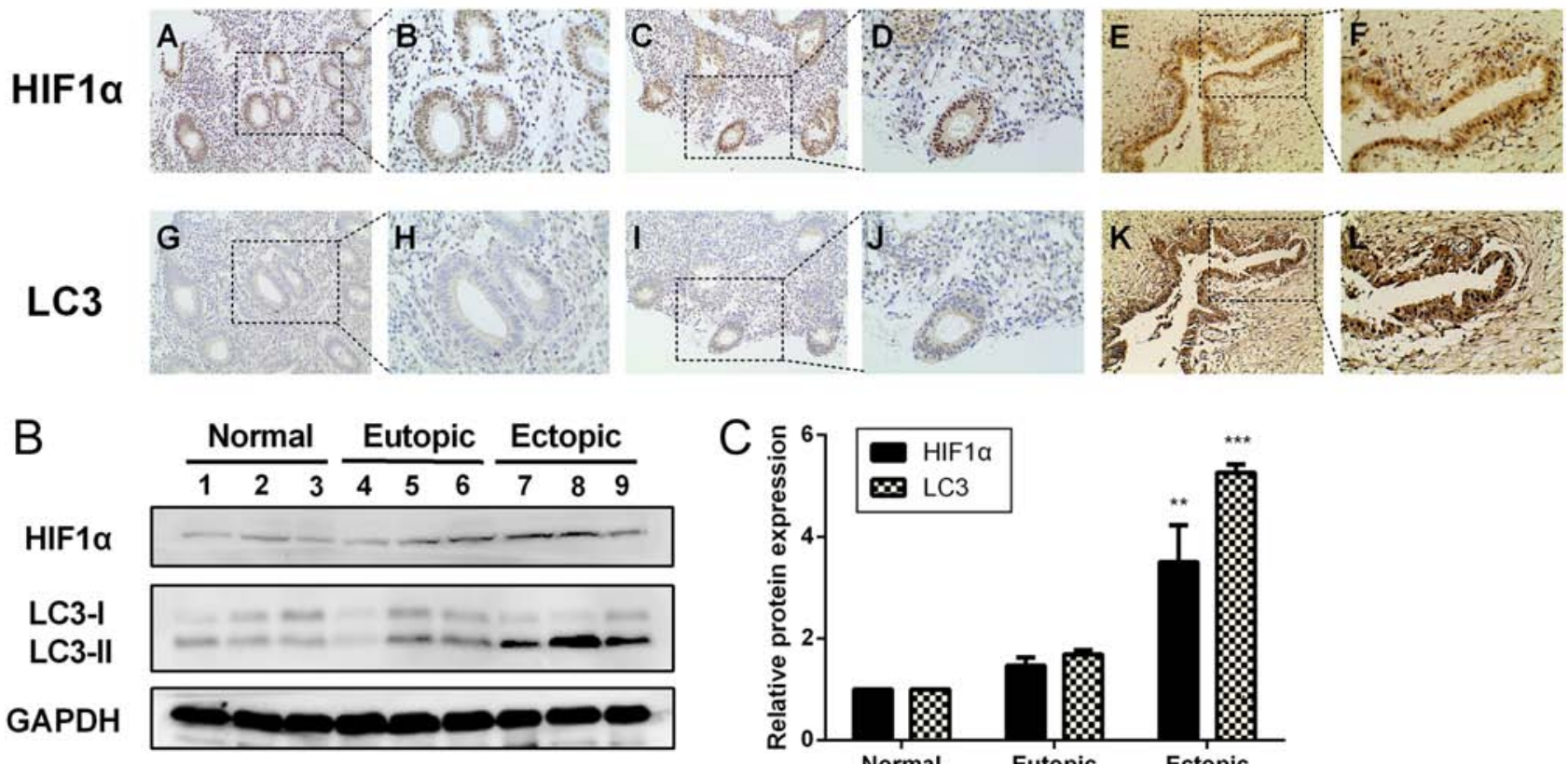

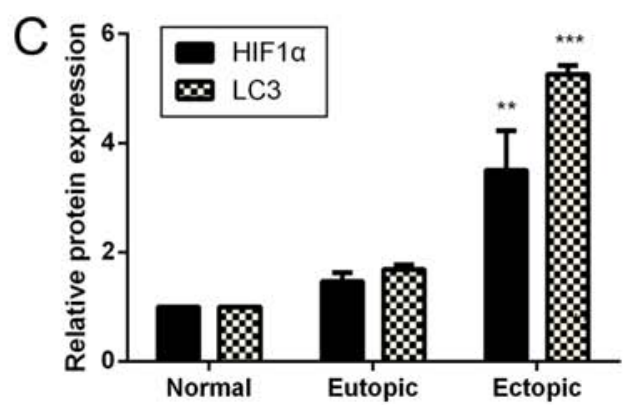

Figure 1 The expression levels of HIF1 $\alpha$ and LC3 protein were elevated in ectopic endometrium of endometriosis patients. (A) Representative immunohistochemical images of HIF- $1 \alpha$ and LC3 protein localization in normal endometrium (A, B, G, H), eutopic endometrium (C, D, I, J) and ectopic endometrium (E, F, K, L). Photographs were taken at magnifications of 200x (left panels) and 400x (right panels) respectively.

(B) Representative Western blots of HIF-1 $\alpha$ and LC3 protein from normal endometrium, eutopic endometrium and ectopic endometrium. (C) The protein expression levels were quantified by the fold difference using Imagej software and normalized to GAPDH protein levels. The data are presented as the means \pm S.D. from at least three independent experiments $\left({ }^{*} P<0.05 ;{ }^{* *} P<0.01 ;{ }^{* * *} P<0.001\right.$ by Student's $t$-test). 

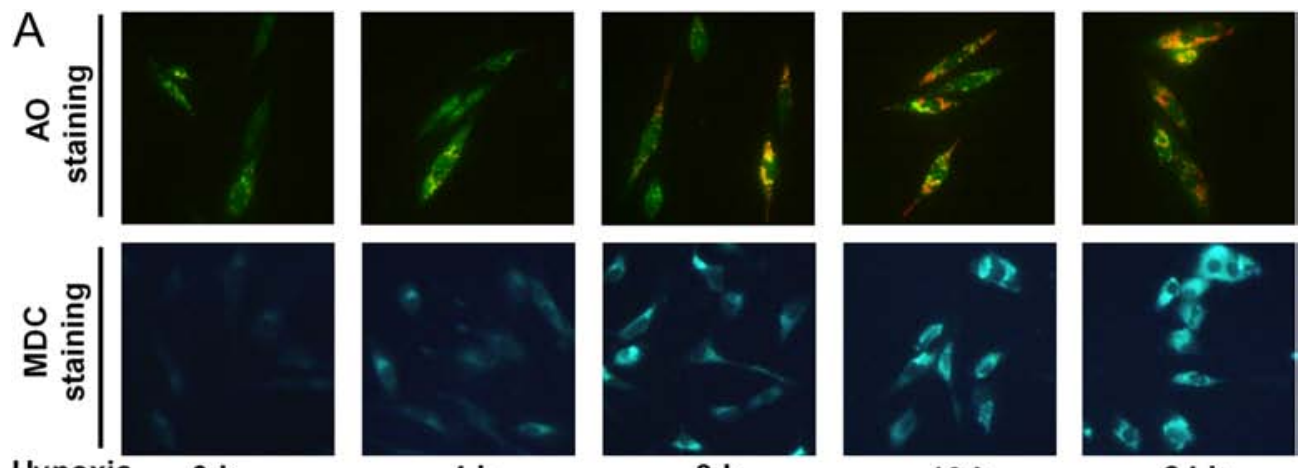

Hypoxia

O h

$4 \mathrm{~h}$

$16 \mathrm{~h}$

$24 \mathrm{~h}$

B

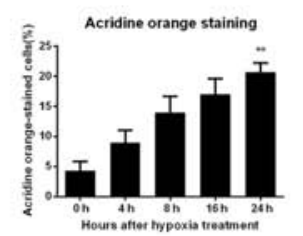

C Normoxia
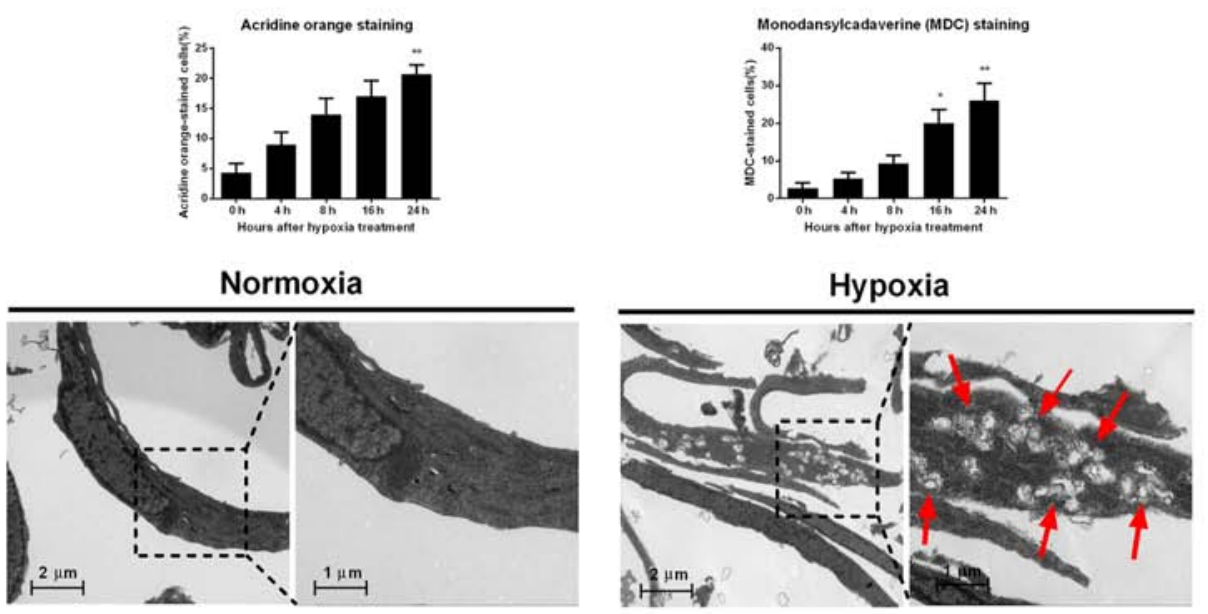

D
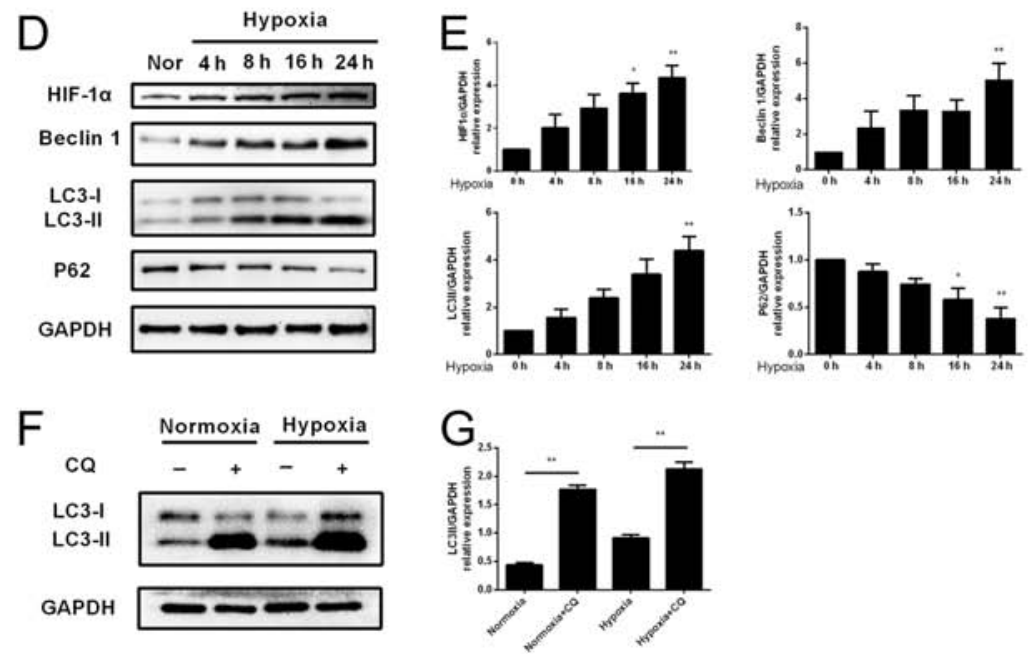

Figure 2 Hypoxia-induced autophagy in HESCs. (A) Representative images of HESCs treated with hypoxia for 0, 4, 8, 16 and 24 h, respectively and then cells were stained with acridine orange and MDC. (B) The percentage of cells stained for acridine orange or MDC in (A) was quantified. The data are presented as the means \pm S.D. from at least three independent experiments $\left({ }^{*} P<0.05 ;{ }^{* *} P<0.01 ;{ }^{* * *} P<0.001\right.$ by the Kruskal-Wallis test). (C) Representative images of transmission electron microscopy performed on HESCs receiving hypoxic treatment as described in the 'Materials and methods' section. Transmission electron microscopy photographs of HESCs cultured under normoxic conditions for $24 \mathrm{~h}$ (left panel) or hypoxia conditions for $24 \mathrm{~h}$ (right panel). The red arrows point to autophagosomes (scale bar, $1 \mu \mathrm{m}$ ). (D) HESCs were incubated under hypoxic conditions for $0,4,8,16$ and $24 \mathrm{~h}$ and then lysed and analyzed by Western blots to determine the expression levels of HIF-1 $\alpha$, Beclin 1 and LC3. (E) The quantitative comparison of the fold difference in the expression of HIF- $1 \alpha$, Beclin 1 and LC3 protein. Total protein levels were normalized to GAPDH levels. The data are presented as the means \pm S.D. from at least three independent experiments $\left({ }^{*} P<0.05 ;{ }^{* *} P<0.01 ;{ }^{* * *} P<0.001\right.$ by the Kruskal-Wallis test). (F) Representative Western blots of LC3 protein under normoxic and hypoxic conditions, in the presence or absence of chloroquine. (G) The expression levels of LC3 protein were quantified by ImageJ software and normalized to GAPDH protein levels. The data are presented as the means \pm S.D. from at least three independent experiments $\left({ }^{*} P<0.05 ;{ }^{* *} P<0.01 ;{ }^{* * *} P<0.001\right.$ by the Kruskal-Wallis test). 
analysis displayed that the protein expression levels of HIF-1 $\alpha$, Beclin1 and LC3-II were increased in a timedependent manner after hypoxia treatment (Fig. 2D and E). As the elevation of LC3 protein level could be resulted from increased autophagosomes formation or decreased autophagosome degradation (Mizushima \& Yoshimori 2007), autophagy flux was evaluated in the presence and absence of lysosomal degradation inhibitor chloroquine. As shown in Fig. $2 \mathrm{~F}$ and $\mathrm{G}, \mathrm{a}$ blockade of the autophagosome-lysosome fusion using chloroquine significantly increased the accumulation of endogenous LC3-II protein, and hypoxia apparently augmented this effect, indicating that hypoxia-induced elevation of LC3-II is due to autophagy activation, rather than blockage of lysosomal degradation. Altogether, these results demonstrate that hypoxia is able to induce autophagy in HESCs.

\section{HIF-1 $\alpha$ overexpression induces autophagy under normoxic condition}

To examine whether HIF- $1 \alpha$ has an effect on autophagy, HESCs were transfected with a HIF- $1 \alpha$ expression plasmid under normoxia conditions. Western blot results showed that HIF- $1 \alpha$ overexpression caused increased expression levels of HIF-1 $\alpha$ and Beclin1 and LC3 (Fig. 3A and B). In addition, we found that compared with the negative control group, the HESCs transfected with HIF-1 $\alpha$ overexpression plasmid showed typically dense accumulation of GFP-LC3 puncta in the perinuclear region under normoxia condition (Fig. 3C).

\section{Knockdown of HIF-1 $\alpha$ interferes with hypoxia-induced autophagy in HESCs}

To further corroborate the role of HIF- $1 \alpha$ in hypoxiainduced autophagy, HESCs were transfected with specific siRNA targeting HIF-1 $\alpha$ under hypoxic conditions. Compared with HESCs transfected with control siRNA, decreased expression of HIF- $1 \alpha$ and Beclin1 and LC3 were observed in HESCs transfected with HIF- $1 \alpha$ siRNA under hypoxia condition (Fig. 4A andB). Moreover, GFP-LC3 puncta accumulation was significantly decreased in HESCs transfected with HIF$1 \alpha$ siRNA compared to that of negative control group under hypoxia condition (Fig. 4C). These, together with the previously mentioned results, suggest that autophagy upregulation under hypoxic condition was dependent on the status of HIF- $1 \alpha$.

\section{Hypoxia enhances the migration and invasion of the HESCs in a HIF-1 $\alpha$-dependent manner}

To explore the effect of hypoxia on cellular motility, transwell migration and invasion assays were conducted. Treatment with hypoxia significantly enhanced the migration and invasion ability of HESCs, when compared
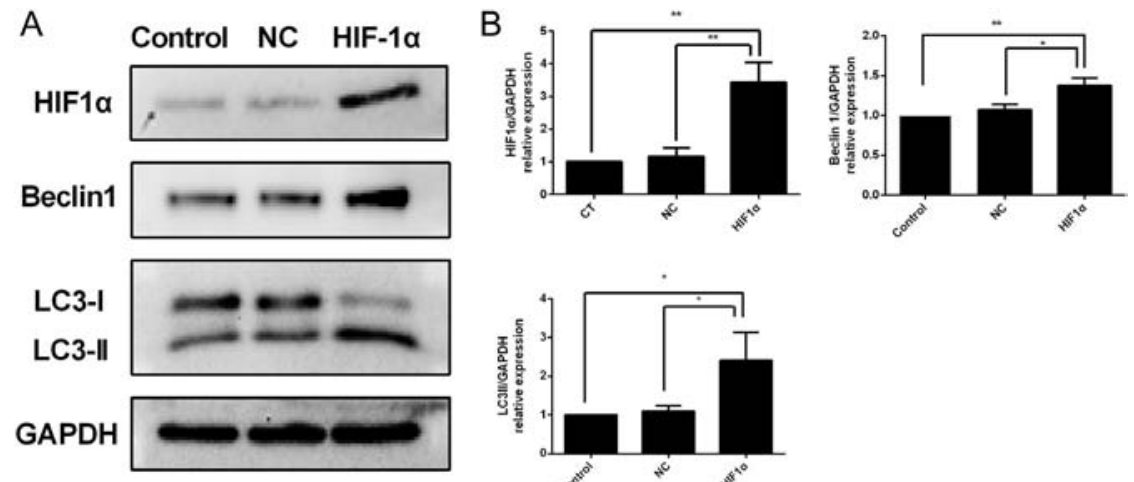

C

LC3
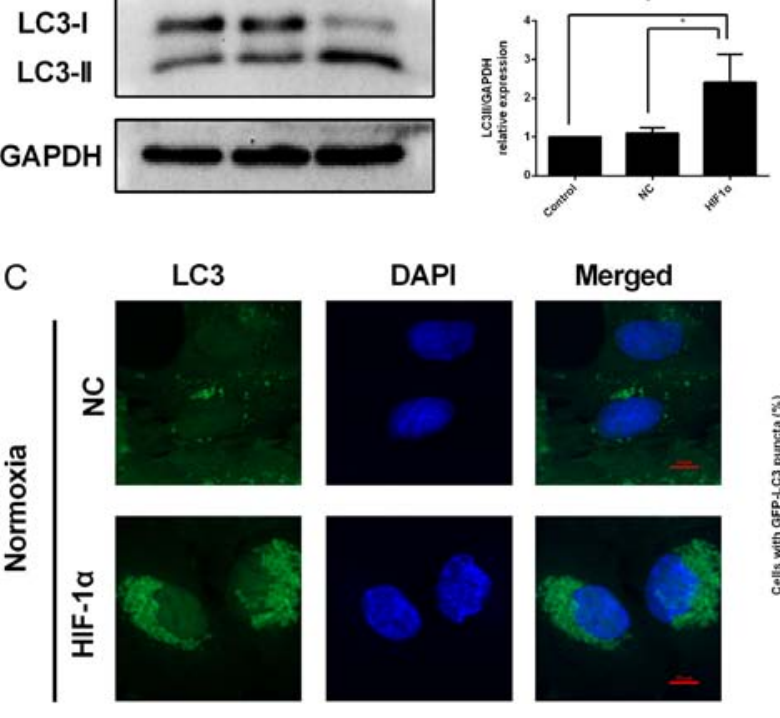

\begin{abstract}
DAPI
\end{abstract}
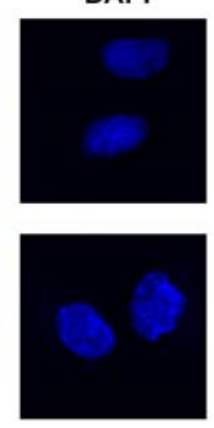

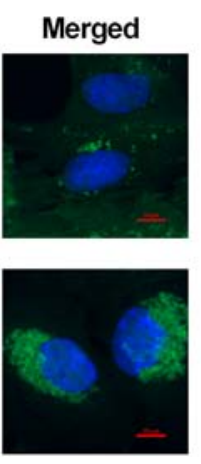

\section{D}

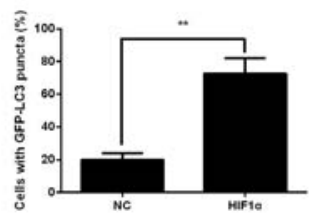

Figure $3 \mathrm{HIF}-1 \alpha$ overexpression induces autophagy under normoxic conditions. (A) Representative Western blots of HIF- $1 \alpha$, Beclin1 and LC3 protein in HESCs transfected with negative control (NC) plasmid or HIF- $1 \alpha$ expression plasmid under normoxic condition. (B) The protein expression levels were quantified by ImageJ software and normalized to GAPDH protein levels. The data are presented as the means \pm S.D. from at least three independent experiments $\left({ }^{*} P<0.05\right.$; ${ }^{* *} P<0.01$; ${ }^{* * *} P<0.001$ by one-way ANOVA). (C) Detection of GFP-LC3 puncta indicative of autophagy in HESCs transfected with negative control (NC) plasmid or HIF-1 $\alpha$ expression plasmid under normoxic condition for $24 \mathrm{~h}$. Nuclei were stained with DAPI. Photographs were taken at magnifications of $1600 \times$. (D) Quantification of the percentage of cells displaying punctate GFP-LC3 using ImageJ. The data are presented as the means \pm S.D. from at least three independent experiments $\left({ }^{*} P<0.05 ;{ }^{* *} P<0.01 ;{ }^{* * *} P<0.001\right.$ by Student's $t$ test). 


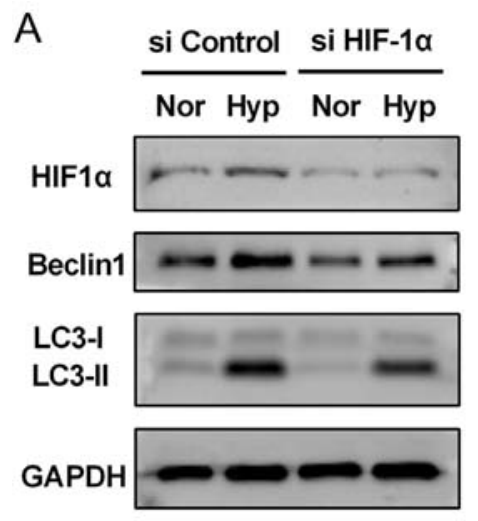

B
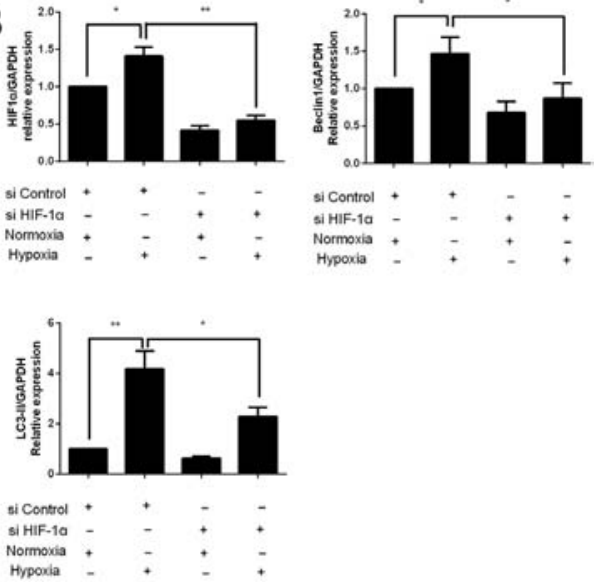

C

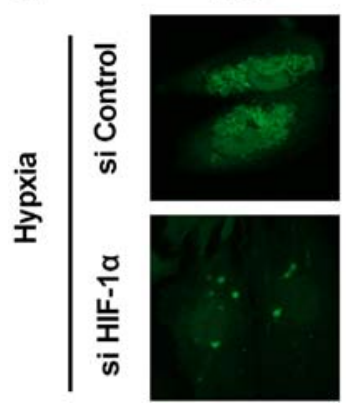

DAPI

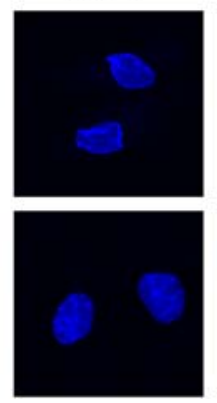

Merged

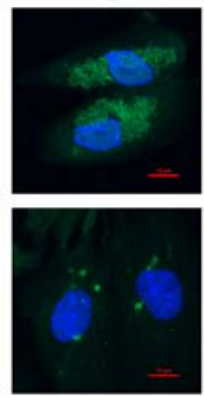

D

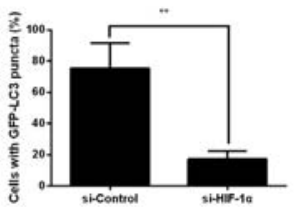

Figure 4 Knockdown of HIF-1 $\alpha$ interferes with hypoxia-induced autophagy in HESCs.

(A) Representative Western blots of HIF- $1 \alpha$, Beclin1 and LC3 protein in HESCs transfected with scrambled control siRNA or HIF- $1 \alpha$ specific siRNA in the presence or absence of hypoxia. (B) The protein expression levels were quantified by ImageJ software and normalized to GAPDH protein levels. The data are presented as the means \pm S.D. from at least three independent experiments $\left({ }^{*} P<0.05\right.$; ** $P<0.01$; *** $P<0.001$ by one-way ANOVA). (C) Detection of GFP-LC3 puncta indicative of autophagy in HESCs transfected with scrambled control siRNA or HIF- $1 \alpha$ specific siRNA under hypoxia condition for $24 \mathrm{~h}$. Photographs were taken at magnifications of 1600×. (D) Quantification of the percentage of cells displaying punctate GFP-LC3 using ImageJ. The data are presented as the means \pm S.D. from at least three independent experiments $\left({ }^{*} P<0.05\right.$; ${ }^{* *} P<0.01$; ***P<0.001 by Student's $t$ test). with the untreated control cells (Fig. 5A and B). On the contrary, the hypoxia-triggered invasive ability could be attenuated by HIF- $1 \alpha$ siRNA. The number of cells that crossed the lower chamber decreased upon
HIF- $1 \alpha$ siRNA treatment (Fig. $5 \mathrm{~A}$ and $\mathrm{B}$ ). These results demonstrated hypoxia can augment the ability of migration and invasion of HESCs in vitro, and this event was dependent on HIF- $1 \alpha$.

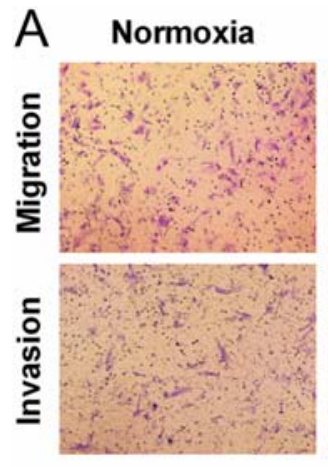

B

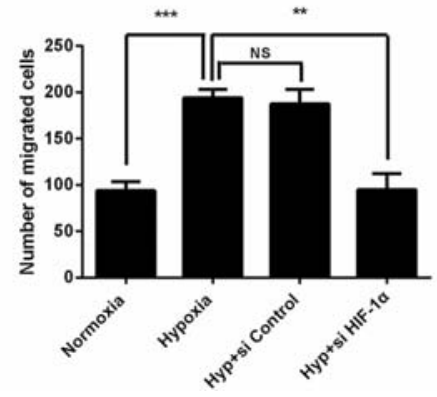

Hypoxia
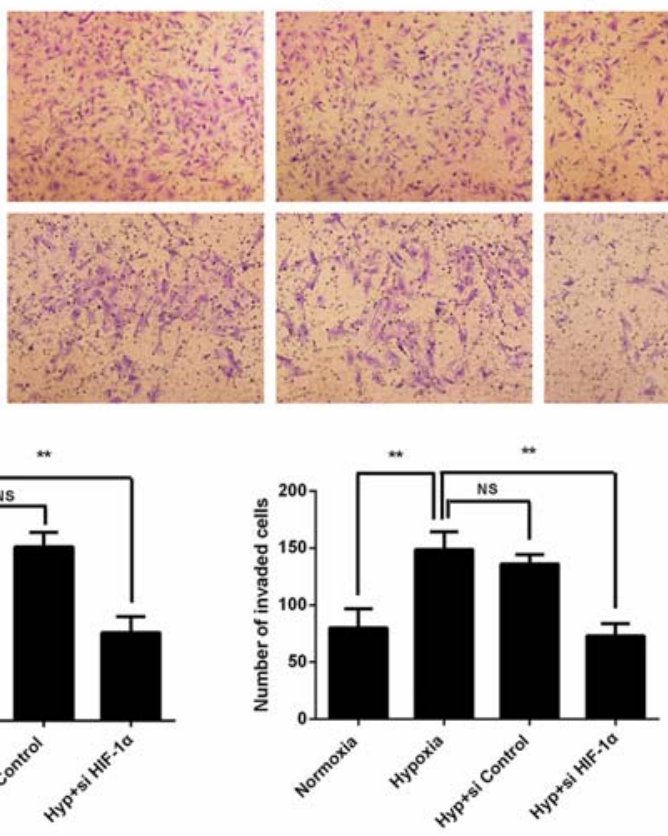

Hyp+si Control Hyp+si HIF-1 $\alpha$

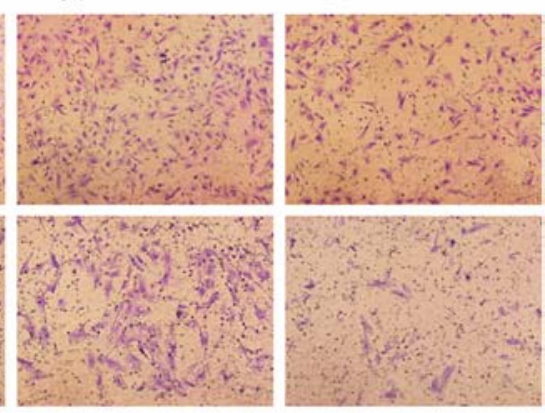

Figure 5 Hypoxia enhances the migration and invasion of the HESCs via HIF-1 $\alpha$. (A) Representative photographs of migration and invasion of hypoxia treated, hypoxia with si-control cotreated or hypoxia with si-HIF- $1 \alpha$ cotreated HESCs. (B) Quantitative assessment of the number of HESCs migrated and invaded to the lower chamber. The data are presented as the means \pm S.D. from at least three independent experiments $\left({ }^{*} P<0.05\right.$; ${ }^{* *} P<0.01 ;{ }^{* * *} P<0.001$ by one-way ANOVA). Photographs were taken at magnifications of $200 x$. 

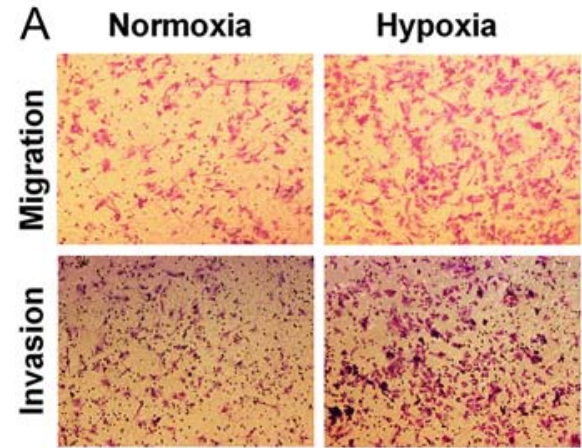

\section{Hyp+3-MA}
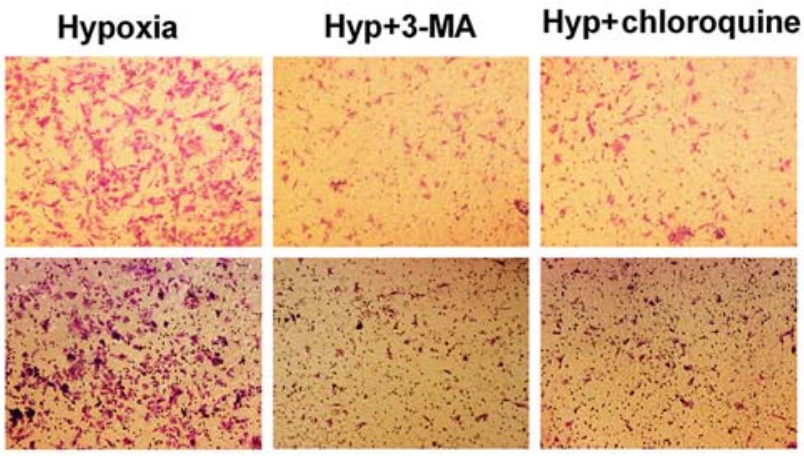

B
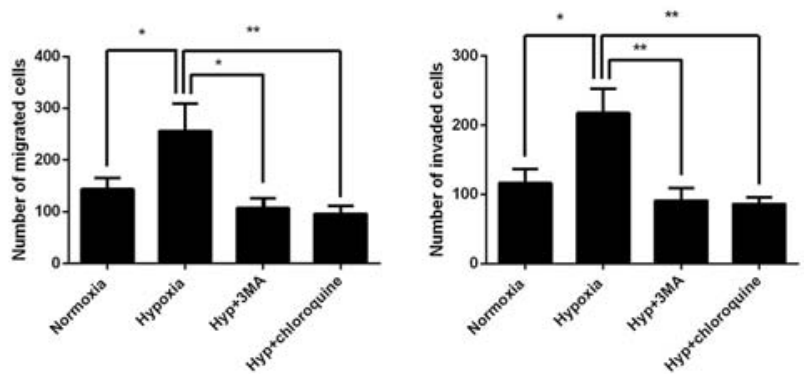

Figure 6 Autophagy inhibition by 3-MA and chloroquine attenuates hypoxia induced HESCs migration and invasion.

(A) Representative photographs of migration and invasion of control, hypoxia treated, hypoxia with 3-MA cotreated or hypoxia with chloroquine cotreated HESCs. (B) Quantitative assessment of the number of HESCs migrated and invaded to the lower chamber. The data are presented as the means \pm S.D. from at least three independent experiments $\left({ }^{*} P<0.05\right.$; ${ }^{* *} P<0.01 ;{ }^{* * *} P<0.001$ by one-way ANOVA). Photographs were taken at magnifications of $200 \times$.

\section{Hypoxia-induced HESCs migration and invasion are attenuated by autophagy inhibition}

To examine whether autophagy has an effect on migration and invasion of HESCs under hypoxia condition, transwell migration and invasion assays were conducted. Here, two types of autophagy inhibitors,
3-MA and chloroquine, were used to inhibit autophagy. 3-MA inhibits autophagy at an early stage by blocking autophagosome formation via the inhibition of type III phosphatidylinositol 3-kinases, involved in the initiation of autophagosome formation (Wu et al. 2010). Chloroquine inhibits autophagy at late stage by inhibiting
A
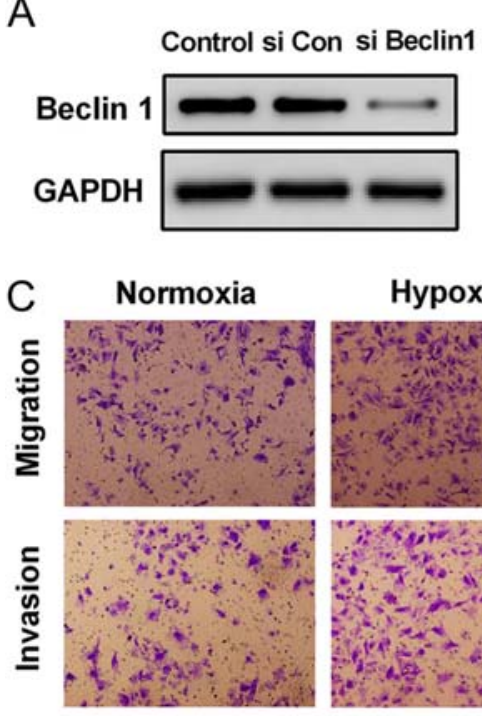

D
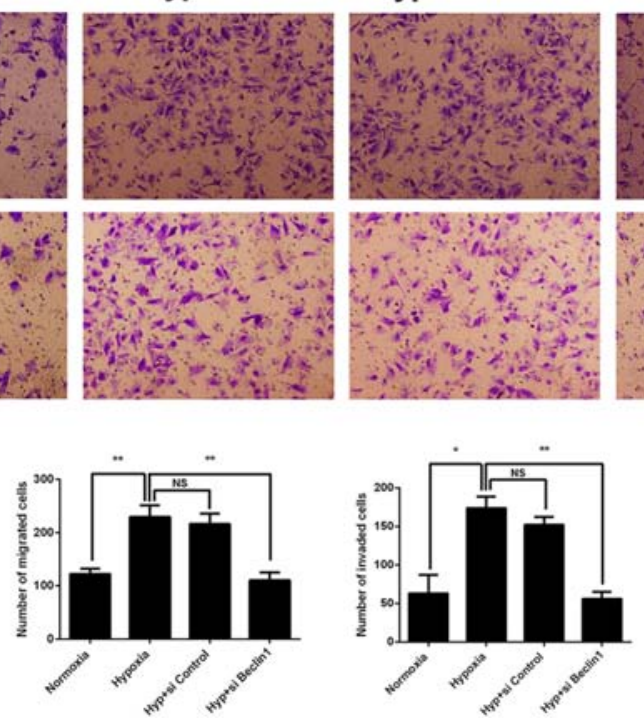
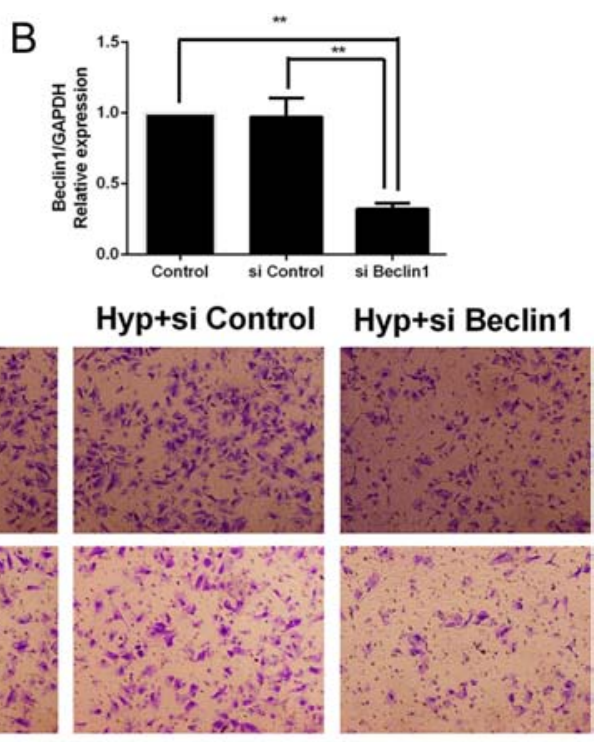

Figure 7 Genetic inhibition of autophagy attenuates hypoxia induced HESCs migration and invasion. (A) Representative Western blots of Beclin1 protein in HESCs transfected with scrambled control siRNA or Beclin1 specific siRNA. (B) The protein expression levels were quantified by ImageJ software and normalized to GAPDH protein levels. The data are presented as the means \pm S.D. from at least three independent experiments $\left({ }^{*} P<0.05\right.$; **P $<0.01 ;{ }^{* * *} P<0.001$ by one-way ANOVA). (C) Representative photographs of migration and invasion of control, hypoxia treated, hypoxia with si-control cotreated or hypoxia with si-Beclin1 cotreated HESCs. (D) Quantitative assessment of the number of HESCs migrated and invaded to the lower chamber. The data are presented as the means \pm S.D. from at least three independent experiments $\left({ }^{*} P<0.05\right.$; ${ }^{* *} P<0.01$; $* * * P<0.001$ by one-way ANOVA). Photographs were taken at magnifications of $200 \times$. 
lysosomal proteases and preventing autophagosomelysosome fusion (Geng et al. 2010). Our data revealed that HESCs treated with hypoxia displayed significantly increased migration and invasion abilities comparison to those untreated, which was nevertheless markedly reversed by the addition of 3-MA or chloroquine (Fig. 6A and B). The pro-invasion role of autophagy in HESCs was further validated by genetically impairing the autophagy pathway using siRNA to Beclin1. As shown in Fig. 7A and B, the Beclin1 siRNA suppressed the expression of the Beclin1 protein. Similar to autophagy inhibition by 3-MA and chloroquine, the genetic inhibition of autophagy by Beclin1 siRNA also attenuated HESCs migration and invasion abilities under hypoxia condition (Fig. 7C and D). Collectively, these results demonstrated that autophagy facilitates the hypoxia-triggered migration and invasion of HESCs in vitro.

\section{Discussion}

Although endometriosis is recognized as a benign disease, its behavior is characterized by certain biological features that also are seen in malignancy (Bassi et al. 2009). The migration, invasion and angiogenesis of viable endometrial tissues outside the uterine cavity is a crucial step for the progression of endometriosis (Moggio et al. 2012). Sampson's retrograde menstruation hypothesis is the most widely accepted theory. However, this theory does not fully explain why most women suffer from retrograde menstruation but only 10 percent of them finally develop endometriosis. Researchers have found that other factors like local hypoxia microenvironment may contribute to the development of endometriosis. When shed endometrial tissue fragments retrograde to the pelvic cavity, the first stress faced is the local altered hypoxic microenvironment. Accumulating evidence reported that HIF-1 $\alpha$ was upregulated in ectopic endometrium and possibly involved in the invasion process of HESCs (Lu et al. 2014, Filippi et al. 2016, Zhan et al. 2016). Therefore, a better understanding of the molecular mechanisms of HIF- $1 \alpha$-regulated endometrial cells migration and invasion is helpful for the treatment of this disease.

Autophagyisimportantinkeeping cellularhomeostasis, and its dysregulation is closely linked to numerous human pathophysiological processes, including cancer, myopathy and neurodegeneration disease (Levine \& Kroemer 2008). Stressful conditions, like hypoxia, can trigger the activation of autophagy (Wu et al. 2015b). Recently, regulation of autophagy by HIF- $1 \alpha$ has been reported. Hypoxia leads to HIF- $1 \alpha$ stabilization, which subsequently activates the downstream gene BNIP3 that competes with $\mathrm{Bcl}-2$ and $\mathrm{Bcl}-\mathrm{XL}$ for interaction with Beclin to trigger autophagy (Bellot et al. 2009). Autophagy is generally considered as a mechanism of cellular protection. In addition, autophagy has also been shown to be involved in modulating cancer cell motility and invasion (Mowers et al. 2016). For example, autophagy could facilitate TLR3- and TLR4-triggered invasion of lung cancer cells (Zhan et al. 2014) and contributes to salivary adenoid cystic carcinoma cells invasion under hypoxia environment (Wu et al. 2015a). In recent years, increasing research efforts have investigated the possible regulatory mechanism of autophagy in hypoxia-triggered cell migration. Autophagy induction during intermittent hypoxia could facilitate the invasiveness of pancreatic cancer cell through the activation of epithelialmesenchymal transition (Zhu et al. 2014). Moreover, autophagy upregulated by HIF-1 $\alpha$ overexpression could support extra-villous trophoblasts invasion by supplementation of cellular adenosine triphosphate (ATP) (Yamanaka-Tatematsu et al. 2013). Interestingly, autophagy activation in MDA-MB-231 cells resulted in attenuated invasiveness through HIF- $1 \alpha$ degradation by autophagic pathway (Indelicato et al. 2010). These apparently disparate conclusions in the field suggests that autophagy may play more complicated roles in tumor invasion, which will be an interesting area for future study.

Previous study indicated that autophagy is upregulated in ovarian endometriosis and possibly contributes to survival of endometriotic cells and to lesion maintenance in ectopic sites (Allavena et al. 2015). However, there have been controversial results regarding the expression level of autophagy in endometriosis. Choi and coworkers reported that autophagy level was decreased in ectopic endometrium together with the activation of p70S6K phosphorylation (signature of mTOR activation) (Choi et al. 2014). These contrasting results may be explained by the fact that a complex signaling network is involved in the regulation of autophagy. The study has found that akt-mammalian target of rapamycin (mTOR) signaling was activated in ovarian endometriosis (Yagyu et al. 2006, Leconte et al. 2011). As a negative regulator of autophagy, mTOR activation may resulted in autophagy inhibition in endometriosis. In fact, besides the canonical PI3K-AKTmTOR signaling (Wu et al. 2009), autophagy can be also induced through non-canonical signaling-like ammonia pathway (Polletta et al. 2015) and hypoxia-inducible factor (HIF)-dependent pathways (Bellot et al. 2009). Based on the previously mentioned correlations between HIF- $1 \alpha$, autophagy and endometriosis, we hypothesized that autophagy upregulation in endometriosis may be due to local hypoxia, and autophagy plays a role in HIF$1 \alpha$-induced HESCs migration and invasion. To elucidate these questions, we designed and conducted a series of investigations.

In our present study, our results from immunohistochemical staining andWestern blots showed that both HIF- $1 \alpha$ - and autophagy-related protein LC3 expression levels were elevated in ectopic endometrium 
compared with normal and eutopic endometrium of endometriosis patients, which indicated that autophagy was upregulated and HIF- $1 \alpha$ may be correlated with this event. After hypoxia treatment for different time points, the protein expression level of HIF- $1 \alpha$, Beclin 1 and LC3 were upregulated. Meanwhile, increased autophagic vacuoles and autophagosome accumulation were observed under hypoxic conditions. To elucidate the regulatory role of HIF- $1 \alpha$ on autophagy, we transfected HESCs with HIF- $1 \alpha$ overexpression plasmid or HIF- $1 \alpha$ siRNA. The results showed that overexpression of HIF$1 \alpha$ resulted in upregulated autophagy under normoxic condition and HIF- $1 \alpha$ siRNA abrogated hypoxia-induced autophagy. Furthermore, to investigate the effect of HIF- $1 \alpha$ and autophagy on cell migration and invasion, transwell assays were performed. We observed that hypoxia was able to enhance migration and invasion of HESCs, whereas transfection with HIF- $1 \alpha$ siRNA reversed this effect, suggesting that hypoxia promotes HESCs cell migration and invasion through HIF-1 $\alpha$. Furthermore, the application of autophagy inhibitors and specific Beclin1 siRNA significantly reversed the hypoxia-stimulated migration and invasion of HESCs.

There are three limitations in the present study: (a) the sample size is relatively small; (b) the expression of autophagy has not been detected in different phases of the menstrual cycle and (c) the exact molecular mechanisms underlying autophagy in HESCs invasion under hypoxia environment remains to be established. Thus, future research is needed to gain deeper insight into these questions.

In conclusion, we demonstrated in this study that HIF- $1 \alpha$ is able to enhance the migration and invasion of HESCs by upregulating autophagy. It is worth noting that autophagy inhibitor chloroquine has been applied to a series of clinical trials targeting malignant diseases like melanoma (Rangwala et al. 2014) and lung cancer (Goldberg et al. 2012). Moreover, a study using murine endometriosis model revealed that inhibition of autophagy by hydroxychloroquine effectively promotes apoptosis of human endometriotic cells and decreases the number of endometriotic lesions (Ruiz et al. 2016). Taken together, these findings reinforce the view that inhibition of autophagy might act as a therapeutic tool in the prevention and treatment of endometriosis.

\section{Supplementary data}

This is linked to the online version of the paper at http://dx.doi. org/10.1530/REP-16-0643.

\section{Declaration of interest}

The authors declare that there is no conflict of interest that could be perceived as prejudicing the impartiality of the research reported.

\section{Funding}

This study was supported by the National Natural Science Foundation of China (Grant No. 81471439 to Y Liu) and the NIH Award (Grant NIH HD076257 to Z B Zhang).

\section{References}

American Fertility Society 1997 Revised American Society for Reproductive Medicine classification of endometriosis: 1996. Fertility and Sterility 67 817-821. (doi:10.1016/S0015-0282(97)81391-X)

Allavena G, Carrarelli P, Del Bello B, Luisi S, Petraglia F \& Maellaro E 2015 Autophagy is upregulated in ovarian endometriosis: a possible interplay with p53 and heme oxygenase-1. Fertility and Sterility 103 1244.e12411251.e1241. (doi.org/10.1016/j.fertnstert.2015.02.007)

Bassi MA, Podgaec S, Dias Júnior JA, Sobrado CW \& Abrão MS 2009 Bowel endometriosis: a benign disease? Revista da Associação Médica Brasileira 55 611-616. (doi:10.1590/S010442302009000500029)

Bellot G, Garcia-Medina R, Gounon P, Chiche J, Roux D, Pouyssegur J \& Mazure NM 2009 Hypoxia-induced autophagy is mediated through hypoxia-inducible factor induction of BNIP3 and BNIP3L via their BH3 domains. Molecular and Cellular Biology 29 2570-2581. (doi:10.1128/ MCB.00166-09)

Bhogal RH, Weston CJ, Curbishley SM, Adams DH \& Afford SC 2012 Autophagy: a cyto-protective mechanism which prevents primary human hepatocyte apoptosis during oxidative stress. Autophagy $\mathbf{8}$ 545-558. (doi:10.4161/auto.19012)

Cheng JC, Klausen C \& Leung PC 2013 Hypoxia-inducible factor 1 alpha mediates epidermal growth factor-induced down-regulation of E-cadherin expression and cell invasion in human ovarian cancer cells. Cancer Letters 329 197-206. (doi:10.1016/j.canlet.2012.10.029)

Choi J, Jo M, Lee E, Kim HJ \& Choi D 2014 Differential induction of autophagy by mTOR is associated with abnormal apoptosis in ovarian endometriotic cysts. Molecular Human Reproduction 20 309-317. (doi:10.1093/molehr/gat091)

Filippi I, Carrarelli P, Luisi S, Batteux F, Chapron C, Naldini A \& Petraglia F 2016 Different expression of hypoxic and angiogenic factors in human endometriotic lesions. Reproductive Sciences 23 492-497. (doi:10.1177/1933719115607978)

Geng Y, Kohli L, Klocke BJ \& Roth KA 2010 Chloroquine-induced autophagic vacuole accumulation and cell death in glioma cells is p53 independent. Neuro-Oncology 12 473-481.

Giudice LC \& Kao LC 2004 Endometriosis. Lancet 364 1789-1799. (doi:10.1016/S0140-6736(04)17403-5)

Goldberg SB, Supko JG, Neal JW, Muzikansky A, Digumarthy S, Fidias P, Temel JS, Heist RS, Shaw AT, McCarthy PO et al. 2012 A phase I study of erlotinib and hydroxychloroquine in advanced non-small-cell lung cancer. Journal of Thoracic Oncology 7 1602-1608. (doi:10.1097/ JTO.0b013e318262de4a)

Hale AN, Ledbetter DJ, Gawriluk TR \& Rucker EB 3rd 2013 Autophagy: regulation and role in development. Autophagy 9 951-972. (doi:10.4161/auto.24273)

Hsiao KY, Chang N, Lin SC, Li YH \& Wu MH 2014 Inhibition of dual specificity phosphatase-2 by hypoxia promotes interleukin-8-mediated angiogenesis in endometriosis. Human Reproduction 29 2747-2755. (doi:10.1093/humrep/deu255)

Hu YL, Jahangiri A, De Lay M \& Aghi MK 2012 Hypoxia-induced tumor cell autophagy mediates resistance to anti-angiogenic therapy. Autophagy $\mathbf{8}$ 979-981. (doi:10.4161/auto.20232)

Indelicato M, Pucci B, Schito L, Reali V, Aventaggiato M, Mazzarino MC, Stivala F, Fini M, Russo MA \& Tafani M 2010 Role of hypoxia and autophagy in MDA-MB-231 invasiveness. Journal of Cellular Physiology 223 359-368. (doi:10.1002/jcp.22041)

Kang R, Zeh HJ, Lotze MT \& Tang D 2011 The Beclin 1 network regulates autophagy and apoptosis. Cell Death and Differentiation 18 571-580. (doi:10.1038/cdd.2010.191)

Karakus S, Sancakdar E, Akkar O, Yildiz C, Demirpence O \& Cetin A 2016 Elevated serum CD95/FAS and HIF-1alpha levels, but not 
Tie-2 levels, may be biomarkers in patients with severe endometriosis: a preliminary report. Journal of Minimally Invasive Gynecology 23 573-577. (doi:10.1016/j.jmig.2016.01.025)

Leconte $M$, Nicco C, Ngo C, Chereau C, Chouzenoux S, Marut W, Guibourdenche J, Arkwright S, Weill B, Chapron C et al. 2011 The mTOR/AKT inhibitor temsirolimus prevents deep infiltrating endometriosis in mice. American Journal of Pathology 179 880-889. (doi:10.1016/j.ajpath.2011.04.020)

Levine B \& Kroemer G 2008 Autophagy in the pathogenesis of disease. Cell 132 27-42. (doi:10.1016/j.cell.2007.12.018)

Lin SC, Wang CC, Wu MH, Yang SH, Li YH \& Tsai SJ 2012 Hypoxiainduced microRNA-20a expression increases ERK phosphorylation and angiogenic gene expression in endometriotic stromal cells. Journal of Clinical Endocrinology and Metabolism 97 E1515-E1523. (doi:10.1210/ jc.2012-1450)

Lu Z, Zhang W, Jiang S, Zou J \& Li Y 2014 Effect of oxygen tensions on the proliferation and angiogenesis of endometriosis heterograft in severe combined immunodeficiency mice. Fertility and Sterility 101 568-576. (doi:10.1016/j.fertnstert.2013.10.039)

Majmundar AJ, Wong WJ \& Simon MC 2010 Hypoxia-inducible factors and the response to hypoxic stress. Molecular Cell 40 294-309. (doi:10.1016/j.molcel.2010.09.022)

Mizushima N \& Yoshimori T 2007 How to interpret LC3 immunoblotting. Autophagy 3 542-545. (doi:10.4161/auto.4600)

Mizushima N, Yoshimori T \& Levine B 2010 Methods in mammalian autophagy research. Cell 140 313-326. (doi:10.1016/j. cell.2010.01.028)

Moggio A, Pittatore G, Cassoni P, Marchino GL, Revelli A \& Bussolati B 2012 Sorafenib inhibits growth, migration, and angiogenic potential of ectopic endometrial mesenchymal stem cells derived from patients with endometriosis. Fertility and Sterility 98 1521.e1522-1530.e1522. (doi. org/10.1016/j.fertnstert.2012.08.003)

Mowers EE, Sharifi MN \& Macleod KF 2016 Autophagy in cancer metastasis. Oncogene 36 1619-1630. (doi:10.1038/onc.2016.333)

Noyes RW, Hertig AT \& Rock J 1975 Dating the endometrial biopsy. American Journal of Obstetrics and Gynecology 122 262-263. (doi:10.1016/S0002-9378(16)33500-1)

Ohsumi Y \& Mizushima N 2004 Two ubiquitin-like conjugation systems essential for autophagy. Seminars in Cell and Developmental Biology 15 231-236. (doi.org/10.1016/j.semcdb.2003.12.004)

Pierzynska-Mach A, Janowski PA \& Dobrucki JW 2014 Evaluation of acridine orange, LysoTracker Red, and quinacrine as fluorescent probes for long-term tracking of acidic vesicles. Cytometry A 85 729-737. (doi:10.1002/cyto.a.22495)

Polletta L, Vernucci E, Carnevale I, Arcangeli T, Rotili D, Palmerio S, Steegborn C, Nowak T, Schutkowski M, Pellegrini L et al. 2015 SIRT5 regulation of ammonia-induced autophagy and mitophagy. Autophagy 11 253-270. (doi:10.1080/15548627.2015.1009778)

Rangwala R, Chang YC, Hu J, Algazy KM, Evans TL, Fecher LA, Schuchter LM, Torigian DA, Panosian JT, Troxel AB et al. 2014 Combined MTOR and autophagy inhibition: phase I trial of hydroxychloroquine and temsirolimus in patients with advanced solid tumors and melanoma. Autophagy 10 1391-1402. (doi:10.4161/auto.29119)

Ruiz A, Rockfield S, Taran N, Haller E, Engelman RW, Flores I, PaninaBordignon P \& Nanjundan M 2016 Effect of hydroxychloroquine and characterization of autophagy in a mouse model of endometriosis. Cell Death and Disease 7 e2059. (doi:10.1038/cddis.2015.361)

Sampson JA 1927 Peritoneal endometriosis due to the menstrual dissemination of endometrial tissue into the peritoneal cavity. American Journal of Obstetrics and Gynecology 14 422-469. (doi:10.1016/S00029378(15)30003-X)
Semenza GL 2009 Regulation of oxygen homeostasis by hypoxia-inducible factor 1. Physiology 24 97-106. (doi:10.1152/physiol.00045.2008)

Swanlund JM, Kregel KC \& Oberley TD 2010 Investigating autophagy: quantitative morphometric analysis using electron microscopy. Autophagy 6 270-277. (doi:10.4161/auto.6.2.10439)

Wu MH, Chen KF, Lin SC, Lgu CW \& Tsai SJ 2007 Aberrant expression of leptin in human endometriotic stromal cells is induced by elevated levels of hypoxia inducible factor-1alpha. American Journal of Pathology 170 590-598. (doi:10.2353/ajpath.2007.060477)

Wu YT, Tan HL, Huang Q, Ong CN \& Shen HM 2009 Activation of the PI3KAkt-mTOR signaling pathway promotes necrotic cell death via suppression of autophagy. Autophagy 5 824-834. (doi:10.4161/auto.9099)

Wu YT, Tan HL, Shui G, Bauvy C, Huang Q, Wenk MR, Ong CN, Codogno P \& Shen HM 2010 Dual role of 3-methyladenine in modulation of autophagy via different temporal patterns of inhibition on class I and III phosphoinositide 3-kinase. Journal of Biological Chemistry 285 10850-10861. (doi:10.1074/jbc.M109.080796)

Wu H, Huang S, Chen Z, Liu W, Zhou X \& Zhang D 2015a Hypoxiainduced autophagy contributes to the invasion of salivary adenoid cystic carcinoma through the HIF-1alpha/BNIP3 signaling pathway. Molecular Medicine Reports 12 6467-6474.

Wu HM, Jiang ZF, Ding PS, Shao LJ \& Liu RY 2015b Hypoxia-induced autophagy mediates cisplatin resistance in lung cancer cells. Scientific Reports 5 12291. (doi:10.1038/srep12291)

Yagyu T, Tsuji Y, Haruta S, Kitanaka T, Yamada Y, Kawaguchi R, Kanayama S, Tanase Y, Kurita N \& Kobayashi H 2006 Activation of mammalian target of rapamycin in postmenopausal ovarian endometriosis. International Journal of Gynecological Cancer 16 1545-1551. (doi:10.1111/j.15251438.2006.00625.x)

Yamanaka-Tatematsu M, Nakashima A, Fujita N, Shima T, Yoshimori T \& Saito S 2013 Autophagy induced by HIF1alpha overexpression supports trophoblast invasion by supplying cellular energy. PLOS ONE 8 e76605. (doi:10.1371/journal.pone.0076605)

Yang Z \& Klionsky DJ 2010 Mammalian autophagy: core molecular machinery and signaling regulation. Current Opinion in Cell Biology 22 124-131. (doi:10.1016/j.ceb.2009.11.014)

Zhan Z, Xie X, Cao H, Zhou X, Zhang XD, Fan H \& Liu Z 2014 Autophagy facilitates TLR4- and TLR3-triggered migration and invasion of lung cancer cells through the promotion of TRAF6 ubiquitination. Autophagy 10 257-268. (doi:10.4161/auto.27162)

Zhan L, Wang W, Zhang Y, Song E, Fan Y \& Wei B 2016 Hypoxia-inducible factor-1 alpha: a promising therapeutic target in endometriosis. Biochimie 123 130-137. (doi:10.1016/j.biochi.2016.01.006)

Zhao XY, Chen TT, Xia L, Guo M, Xu Y, Yue F, Jiang Y, Chen GQ \& Zhao KW 2010 Hypoxia inducible factor-1 mediates expression of galectin-1: the potential role in migration/invasion of colorectal cancer cells. Carcinogenesis 31 1367-1375. (doi:10.1093/carcin/bgq116)

Zhu H, Wang D, Zhang L, Xie X, Wu Y, Liu Y, Shao G \& Su Z 2014 Upregulation of autophagy by hypoxia-inducible factor-1alpha promotes EMT and metastatic ability of CD133+ pancreatic cancer stem-like cells during intermittent hypoxia. Oncology Reports 32 935-942. (doi:10.3892/ or.2014.3298)

Received 28 November 2016

First decision 9 January 2017

Revised manuscript received 7 March 2017

Accepted 24 March 2017 